\title{
Modeling Transmission Dynamics of COVID-19 in Nepal
}

\author{
Gauri Bhuju1,2, Ganga Ram Phaijoo², Dil Bahadur Gurung² \\ ${ }^{1}$ Department of Mathematics, Bhaktapur Multiple Campus, Bhaktapur, Nepal \\ ${ }^{2}$ Department of Mathematics, School of Science, Kathmandu University, Dhulikhel, Nepal \\ Email: gauri.bhuju@student.ku.edu.np
}

How to cite this paper: Bhuju, G., Phaijoo, G.R. and Gurung, D.B. (2020) Modeling Transmission Dynamics of COVID-19 in Nepal. Journal of Applied Mathematics and Physics, 8, 2167-2173.

https://doi.org/10.4236/jamp.2020.810162

Received: September 1, 2020

Accepted: October 19, 2020

Published: October 22, 2020

Copyright $\odot 2020$ by author(s) and Scientific Research Publishing Inc. This work is licensed under the Creative Commons Attribution International License (CC BY 4.0).

http://creativecommons.org/licenses/by/4.0/

\begin{abstract}
A novel coronavirus disease (COVID-19) is an infectious viral disease caused by SARS-CoV-2. The disease was first reported in Wuhan, China, in December 2019, and it has been epidemic in more than 110 countries. The first case of COVID-19 was found in Nepal on 23 January, 2020. Now the number of confirmed cases is increasing day by day. Thus, the disease has become a major public health concern in Nepal. The propose of this study is to describe the development of outbreak of the disease and to predict the outbreak in Nepal. In the present work, the transmission dynamics of the disease in Nepal is analyzed mathematically with the help of SIR compartmental model. Reported data from June $1^{\text {st }}$ to June $17^{\text {th }} 2020$ of Nepal are used to identify the model parameters. The basic reproduction number of COVID-19 outbreak in Nepal is estimated. Predictions of the peak epidemic time and the final size of the epidemic are made using the model. Our work predicts that, after 125 days from June 1 the infection will reach the peak. In this work, a good correlation between the reported data and the estimation given by our model is observed.
\end{abstract}

\section{Keywords}

COVID-19, Compartmental Model, Basic Reproduction Number, Nepal, Parameter Estimation

\section{Introduction}

COVID-19 was first reported in December 2019 in Wuhan city of China, and later it also became rapidly epidemic in other countries. On 17 June 2020, more than 8.4 million cases have been reported from more than 110 countries and territories. More than 452 thousand deaths and more than 4.24 million people have recovered [1]. The latest data of COVID-19 cases show that the cases are 
increasing rapidly in Nepal.

The first imported case of coronavirus was reported in Nepal on 23 January 2020, from a person traveling from Wuhan, China [2] [3]. There are total 7177 infected people in Nepal from 73 districts out of 77 district on $17^{\text {th }}$ June, 2020; age ranged from 2-month infants to 81-year-old women [3]. Government of Nepal announced country-wide lockdown from 24 March to 21 July to control the disease.

Several mathematical research works have been made to study the transmission dynamics of infectious diseases [4] [5] [6] [7] [8]. Compartmental epidemic models [9] [10] [11] [12] have been proposed to predict future COVID-19 cases, to study its transmission dynamics, and so on. Y. Souleiman et al. predicted the outbreak of COVID-19 in Djibouti with the help of mathematical model [13]. Y. Tang and S. Wang investigated the outbreak of COVID-19 in US [10].

In the present work, a simple epidemic SIR model is taken to track the outbreaks of COVID-19 in Nepal. At first we estimate some model parameters from the data provided by the Government of Nepal and we define the basic reproduction number. Then, using estimated parameters in the model we identify the peak of the outbreak and predict the infected population at the end of infection when no control efforts are implemented. The sources of COVID-19 cases data are taken from [3].

The outbreak of COVID-19 in Nepal has gone through different phases. In the beginning of the first week of May, the total cumulated cases were less than 100 . In the last week, the total number of reported cases was increased to over 1000 and the number went up in June continuously and reached 7177 reported positive cases on June 17. Therefore, our modeling work started from $1^{\text {st }}$ June.

\section{Materials and Methods}

\subsection{Data}

The study is based on the daily reported cases extracted from the Ministry of Health and Population of Nepal [3]. The data contains suspected cases, new confirmed cases, cumulative confirmed cases, recovered cases, and death cases due to COVID-19 infection (Figure 1). From the first week of June, new confirmed cases have been more than 200 . Thus, we choose June $1^{\text {st }}$ to June $17^{\text {th }} 2020$ as the observation date and use the daily reported cumulative and new conform cases (Figure 2).

\subsection{Model Formulation}

For the formulation of the model, the total population at time $t$ is denoted by $N(t)$, it is subdivided into three compartments: Susceptible: $S(t)$, Infected: $I(t)$, and Recovered: $R(t)$. Thus, $N(t)=S(t)+I(t)+R(t)$. It is assumed that there is no birth, no immigration, and recovery from the disease confers immunity against the disease. Also there is a fixed infection rate per day and a fixed recovery time. People are well mixed. 


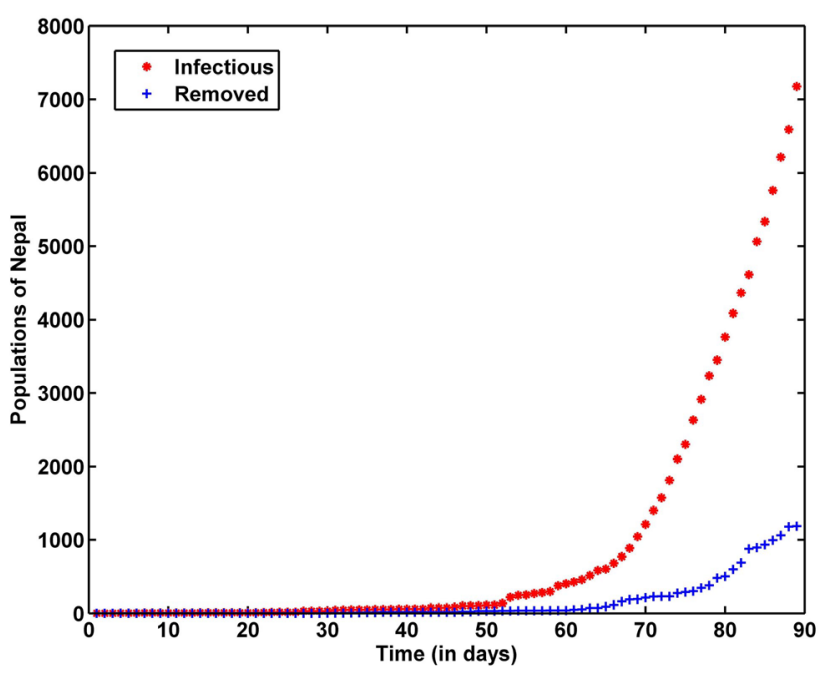

Figure 1. Reported cumulated cases of COVID-19 in Nepal (Mar 23 to Jun 17).

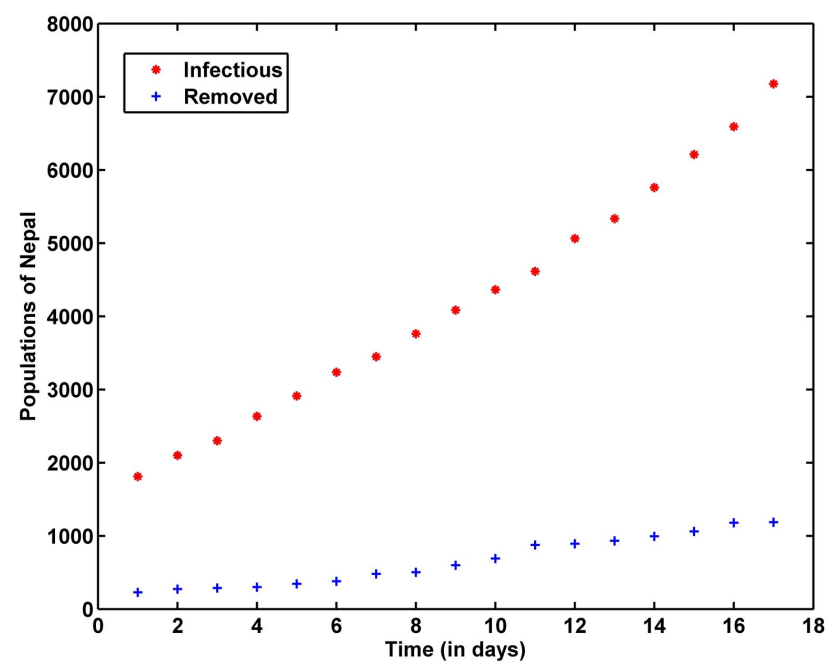

Figure 2. Reported cumulated cases of COVID-19 in Nepal (Jun 1 to Jun 17).

In the present model, the susceptible individuals get infected of COVID-19 when they come in contact with the infectious individuals and they either die or recover from the disease.

$$
\begin{aligned}
& \frac{\mathrm{d} S}{\mathrm{~d} t}=-\frac{\beta}{N} S I \\
& \frac{\mathrm{d} I}{\mathrm{~d} t}=\frac{\beta}{N} S I-\gamma I \\
& \frac{\mathrm{d} R}{\mathrm{~d} t}=\gamma I \\
& N=S+I+R
\end{aligned}
$$

We consider that $\beta$ represents the transmission rate of infectious individuals contacting with $S(t)$ and $\gamma$ represents the removal rate (that is, recovery or death rate) from infection of disease. We do not consider the quarantine and RDT-PCR tested population. 
By re-scaling the state variables with

$$
s=\frac{S}{N}, i=\frac{I}{N}, r=\frac{R}{N}
$$

we get,

$$
\begin{aligned}
& \frac{\mathrm{d} s}{\mathrm{~d} t}=-\beta s i \\
& \frac{\mathrm{d} i}{\mathrm{~d} t}=\beta s i-\gamma i \\
& \frac{\mathrm{d} r}{\mathrm{~d} t}=\gamma i
\end{aligned}
$$

An epidemic occurs if the number of infection increases, that is

$$
\begin{aligned}
& \frac{\mathrm{d} i}{\mathrm{~d} t}>0 \\
\Rightarrow & \beta s i-\gamma i>0 \\
\Rightarrow & \frac{\beta s i}{\gamma}>i
\end{aligned}
$$

At the outset of the epidemic, we have $s \sim 1$

$$
\frac{\beta}{\gamma}>1
$$

$R_{0}$ is the basic reproduction number of the epidemic. Basic reproduction number is defined as the average number of secondary infections caused by a single infectious individuals during their entire infectious life time [14] [15]. Thus we have [14]

$$
\begin{gathered}
R_{0}=(\text { Rate of secondary infection }) \times(\text { duration of infection }) \\
\Rightarrow R_{0}=\beta \times \frac{1}{\gamma} \\
\Rightarrow R_{0}=\frac{\beta}{\gamma}>1
\end{gathered}
$$

We use the Least Square Method to fit best-line fit in COVID-19 data from June 1 to June 17, 2020 of Nepal [3]. After estimating the values of $\beta$ and $\gamma$, we get the basic reproduction number

$$
R_{0}=2.72
$$

In general, initially the infection of disease $i$ grows exponentially as in Figure 3. Then reach the peak, then gradually decay back to 0 . We have

$$
\begin{gathered}
\frac{\mathrm{d} i}{\mathrm{~d} t}=i(\beta s-\gamma) \\
\Rightarrow i=1-s+\frac{\gamma}{\beta} \ln s
\end{gathered}
$$

Since, at the peak $\frac{\mathrm{d} i}{\mathrm{~d} t}=0$, from (3) 


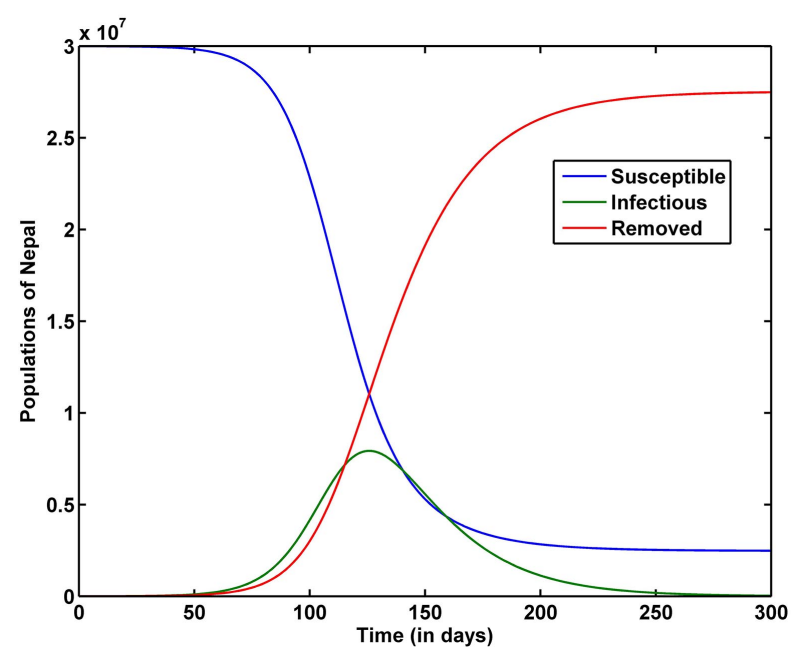

Figure 3. State variables of SIR model of COVID-19 in Nepal.

$$
\Rightarrow s=\frac{\gamma}{\beta}
$$

Putting the value of $s$ in (4), we get

$$
i_{\max }=1+\frac{\gamma}{\beta}\left(\ln \frac{\gamma}{\beta}-1\right)
$$

Using COVID-19 data from June 1 to June 17, 2020 in Nepal [3], we get

$$
i_{\max }=0.2643
$$

Therefore, $26 \%$ get sick at the peak.

At the end of infection, we know that $i=0$ as $t \rightarrow \infty$. Thus, Equation (4) becomes

$$
1-s+\frac{\gamma}{\beta} \ln s=0
$$

On solving, we obtain

$$
S_{\infty}=0.047
$$

Thus, $0.47 \%$ of the population remain susceptible at the end of epidemic. Therefore, $99.53 \%$ get infected at the same time if any control measures does not use.

\section{Result}

In this section, we use the model (1) to fit the cumulative infectious cases from June $1^{\text {st }}$ to June $17^{\text {th }}, 2020$ in Nepal. Through some rational assumptions and parameter estimations, the fitting curves of cumulative cases using model (1) are shown in Figure 4. The simulation of the model is made by numerical solution applying Runge Kutta method of order 4. The figure indicates that our model provides a well fit to the reported data from June $1^{\text {st }}$ to June $17^{\text {th }} 2020$ in Nepal. Figure 4 shows that the disease will grow rapidly in the coming days until it reached to the peak and end of June, there will be about 20,000 infected population in Nepal. 


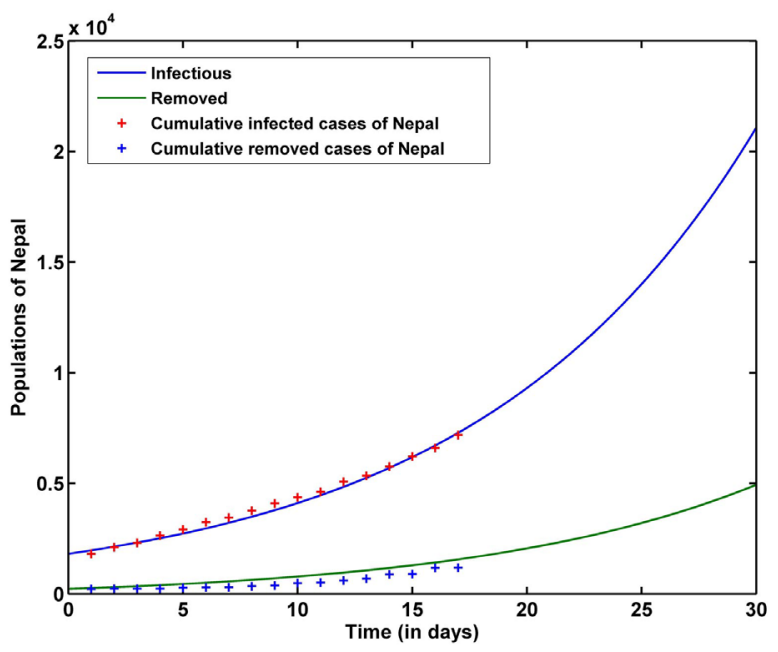

Figure 4. Cumulative confirmed cases and predicted cases by the model.

\section{Conclusions}

Based on the transmission dynamics of COVID-19 in Nepal, we built the timedependent simple mathematical model of COVID-19. Mathematical modeling plays an important role to predict how an outbreak is developing and where it may go and in what kind of time framework. Such types of informations are very critical to control the outbreak, utilize the resources, and to return the normal daily life of citizen of a country.

We use our model to fit the cumulative confirmed cases in Nepal from June $1^{\text {st }}$ to June $17^{\text {th }} 2020$ and get a reasonable match. The basic reproduction number is estimated $R_{0}=2.72$ based on the outbreak data which growth exponentially. Later on, we estimated the maximum number of infection at the peak $i_{\max }=0.2643$, and susceptible population as $S_{\infty}=0.047$, if any control measures are not used. The result shows that the infection will reach at the peak after 125 days from June $1^{\text {st }} 2020$ (From Figure 3). Effective control measures such as social distancing, self-isolation, disease testing facilities, face mask wearing, related policies etc. are helpful in the control of the disease.

\section{Conflicts of Interest}

The authors declare no conflicts of interest regarding the publication of this paper.

\section{References}

[1] World Health Organization (2020) Situation Update, View Coronavirus Disease 2019 (COVID-19). World Health Organization, Geneva.

[2] Bastola, A., Sah, R., Morales, A.J.R. and Chu, D. (2020) The First 2019 Novel Coronavirus in Nepal. National Library of Medicine, 20, 279-280.

https://doi.org/10.1016/S1473-3099(20)30067-0

[3] MOHP (2020) Nepal's Latest Update on COVID-19. Ministry of Health and Population, Kathmandu, Nepal. 
[4] Bhuju, G., Phaijoo, G.R. and Gurung, D.B. (2018) Mathematical Study on Impact of Temperature in Malaria Disease Transmission Dynamics. Advances in Computer Sciences, 1, 107.

[5] Phaijoo, G.R. and Gurung, D.B. (2017) Mathematical Model of Dengue Disease Transmission Dynamics with Control Measures. Journal of Advances in Mathematics and Computer Science, 23, 1-12.

[6] Kermack, W.O. and McKendrick, A.G. (1991) Contribution to the Mathematical Theory of Epidemic. Bulletin of Mathematical Biology, 53, 33-55. https://doi.org/10.1016/S0092-8240(05)80040-0

[7] Phaijoo, G.R. and Gurung, D.B. (2018) Sensitivity Analysis of SEIR-SEI Model of Dengue Disease. GAMS Journal of Mathematics and Mathematical Bioscience, 6, 41-50.

[8] Vaidya, N.K., Li, X. and Wang, F.B. (2019) Impact of Spatially Heterogeneous Temperature on the Dynamics of Dengue Disease. Discrete and Continuous Dynamical System Series B, 10, 1-29.

[9] Koroler, I. (2020) Identification and Estimation of the SEIRD Epidemic Model for COVID-19. Journal of Econometrics.

[10] Tang, Y. and Wang, S. (2020) Mathematic Modeling of COVID-19 in the United States. Emerging Microbes and Infections, 9, 827-829.

[11] Wang, L.P., Wang, J., Zhao, H.Y., Shi, Y.Y., Wang, K., Peng, W. and Shi, L. (2020) Modelling and Assessing the Effects of Medical Resources on Transmission of Novel Coronavirus (COVID-19) in Wuhan, China. Mathematical Bioscience and Engineering, 17, 2936-2949.

[12] Wang, M. and Qi, J.T. (2020) A Deterministic Epidemic Model for the Emergence of COVID-19 in China. MedRxiv, the Preprint Sever for Health Science.

[13] Souleiman, Y., Mohamed, A. and Ismail, L. (2020) Analysis the Dynamics of SIHR Model: COVID-19 Case in Djibouti. Mathematics and Computer Science, Applied Mathematics. https://doi.org/10.20944/preprints202006.0210.v1

[14] Diekmann, O., Heesterbeek, J.A.P. and Metz, J.A.J. (1990) On the Definition and Computation of Basic Reproduction Ratio $R_{0}$ in Models for Infectious Diseases in Heterogeneous Populations. Journal of Mathematical Biology, 28, 365-382. https://doi.org/10.1007/BF00178324.

[15] Van Den Driessche, P. and Watmough, J. (2002) Reproduction Number and SubThreshold Endemic Equilibria for Compartmental Models for Disease Transmission. Mathematical Bioscience, 180, 29-48. https://doi.org/10.1016/S0025-5564(02)00108-6 